\title{
Implicazioni della formula di Clairaut relative al Geoide (Clairaut formula implicatons relating to the Geoid)
}

\author{
C. BERNASCONI *
}

Ricevuta il 20 aprile 1978

\section{RIASSUNTO}

L'Autore osserva che la relazione di Clairaut fra lo schiacciamento $x$ del geoide, il rapporto « fra i momenti di inerzia e la velocità angolare (1), può essere utilizzata secondo l'interpretazione da lui stesso data di 16. per dedurre i momenti di inerzia $I_{\vec{r}}$ ed $I_{\bar{i} \overline{\bar{y}}}$.

Successivamente, riprendendo un precedente lavoro, calcola la variazione di energia gravitazionale nel passaggio da un ipotetico geoide sferico in quiete al geoicle attuale ruotante attorno all'asse polare e ricava il valore $W_{g i}$ della energia dovuta al campo di gravità interno al geoide.

Utilizza poi i dati così ricavati (oltre alla conoscenza sperimentale del valore della densità superficiale del geoide) per uno studio teorico della distribuzione della densità in funzione del raggio e ricerca in particolare, fra i possibili valori della densità al centro, quella di valore minimo.

\section{AbSTRACT}

The Author points out the connection of Clairaut between the flattening $\alpha$ of the geoid, the ratio $u$ of the moments of inertia and the angular speed (1) can be utilized, according to his own interpretation of

* Libero docente di Geodesia - Università di Genova. 
$\mathcal{H}$ in order to deduce the moment of inertia $I_{\vec{F}}$ and $I_{\overline{-}}$. Afterwards, resuming a former work, he calculates the change of gravitational energy in the passing from a hypothetical spherical geoid in rest to the present geoid turning around the polar axis, and he derives the value $W_{\overline{5} \text { i }}$ of the energy due to the gravitational field inside the geoid. Then he makes use of the data thus obtained (as well as the experimental knowledge of the value of the surface density of the geoid) for a theoretical study of the distribution of the density proporionate to the radius, and, particularly, he seeks among the possible values of the density in the minimal density.

Implicazioni della formula Clairaut Relativa al Geoide

Dalla teoria del potenziale applicata al geoide terrestre si ricava la nota formula

$$
x=\frac{3}{2} \frac{C^{2} \|}{2 a^{2}}+\frac{w^{2} a^{2}}{2 \varepsilon M}
$$

con $M=$ Massa del geoide;

$a$ = Semiasse equatoriale;

$w=$ Velocità angolare di rotazione del geoide;

$\varepsilon=$ Costante di attrazione universale.

Detti poi

$$
I_{p}=C^{2} M
$$

il momento d'inerzia rispetto all'asse polare e

$$
I_{c}=A^{2} M
$$

il momento d'inerzia rispetto ad un asse equatoriale, si intende per $\mu$ il rapporto

$$
\mu=\frac{\left(I_{p}-I_{\varepsilon}\right)}{I_{p}}=\frac{\left(C^{2}-A^{2}\right)}{C^{2}}
$$


I valori numerici nel sistema C.G.S. di alcune delle grandezze considerate sono

$$
\begin{gathered}
x=\frac{1}{297}=3,367 \cdot 10^{-3} \quad a=6,37838 \cdot 10^{8} \equiv=6,66 \cdot 10^{-3} \\
w=7,2782 \cdot 10^{-5} ; \frac{w^{2} a^{2}}{\varepsilon M}=\frac{w^{2} a}{g_{\mathrm{o}}}=3,44204 \cdot 10^{-3}
\end{gathered}
$$

essendo $g_{0}=980$ valore medio della gravità alla superficie del geoide.

Se si suppone che il geoide sia un ellissoide omogeneo di rotazione, di semiassi

$$
a \text { e } b=a(1-\alpha)
$$

allora si avrebbe esattamente

$$
\mu=\alpha-\frac{\alpha^{2}}{2}-\alpha
$$

Se si fa l'ipotesi che in ogni caso il geoide sia equiparabile ad un ellisoide la cui densità interna vari come quella del geoide, e in più che il rapporto fra i momenti d'inerzia definito da $u$ sia praticamente indipendente dalla variazione della densità allora nella [1] si può sostituire $\mu \operatorname{con} \alpha$, e sostituendo altresì $C^{2}$ con $I_{p} / M$ e $a^{2} / M$ con $\varepsilon a / g_{0}$ la stessa diviene

$$
\alpha=\frac{3}{2} \frac{\varepsilon}{a^{4} g_{\mathrm{o}}} I_{\mathrm{n}} \alpha+\frac{w^{2} a}{2 g_{0}}
$$

dalla quale si può ricavare $I_{p}$. 
Coi dati numerici precedenti si ha

$$
I_{p}=7,937514 \cdot 10^{44} \quad I_{e}=7,910788 \cdot 10^{44}
$$

In effetti è facile verificare che, data una serie (o un continuo) di ellissoidi di rotazione definiti dai semi-assi $a \cdot \beta_{\mathrm{i}}, a \cdot \beta_{\mathrm{i}}(1-\alpha)$ con $0<r_{i}<1$, ammesso che sia costante la densità di ciascuna fascia compresa fra gli ellissoidi $\beta_{i}$ e $\bar{\beta}_{i+1}$ e che tale densità vari da una fascia all'altra, si ha sempre $\mu=\alpha-\alpha^{2} / 2$

Lo schiacciamento del geoide deriva, come noto, da moto di rotazione. In un precedente articolo l'A. (1974), dopo aver premesso che se si arrestasse il moto di rotazione il geoide diverrebbe sferico e di volume inferiore all'attuale, ha ottenuto una soddisfacente determinazione di $\alpha$, partendo dal principio che la perdita dell'energia cinetica doveva ritrovarsi in un incremento di energia gravitazionale. Il principio è stato tradotto nell'equazione

$$
16 \alpha^{2}-30 x \theta-135 \theta^{2}
$$

essendo $\theta=W_{c} / W_{k}$, dove $W_{c}$ è l'energia cinetica e $W_{g}$ l'energia gravitazionale.

Per comodità del lettore riportiamo gli sviluppi che hanno condotto alla [5].

Ai fini del calcolo è preferibile il procedimento inverso di quello, più intuitivo, accennato nel testo, ossia immaginare che al geoide sferico in quiete si imprima una rotazione di velocita angolare $W$ da ritenersi costante in tutte le successive evoluzioni del geoide ed avente il valore attuale $W=7,2782 \cdot 10^{-5}$.

Il calcolo, che prescinde da ogni considerazione di carattere meccanico ed è basato unicamente sul principio della conservazione dell'energia e della massa, si esegue in due tempi sia quando si prende in considerazione il variare dell'energia gravitazionale, sia quando l'energia cinetica. 


\section{ENERGIA GRAVITAZIONALE}

1) Diciamo $W_{k 1}$ l'energia gravitazionale di una sfera in quiete della stessa massa $M$ del geoide e di volume inferiore a quello dell'attuale ellissoide; sia $R$ il suo raggio. Come noto si ha

$$
W_{g 1}=\frac{3}{5} \frac{M^{2} \varepsilon}{R}=\frac{3}{5} \frac{g_{0}{ }^{2} R^{3}}{\varepsilon} \quad g_{0}=\frac{\varepsilon M}{R^{2}}
$$

Nel gonfiarsi della sfera, il cui raggio passa da $R$ a $R^{3}=R(1+\theta)$ fino ad acquistare il volume dell'attuale ellssoide, l'energia di gravità diminuisce e diventa

$$
W_{a^{2}}=\frac{3}{5} \frac{M^{2} \varepsilon}{R(1+\theta)} \sim \frac{3}{5} \frac{M^{2} \varepsilon}{R}\left(1-\theta+\theta^{2}\right)
$$

2) Nel passare dalla sfera di raggio $R^{3}$ all'ellissoide l'energia ancora diminuisce. Per valutarne l'importo si parte dal rilevare che il valore medio della gravità $g_{0}{ }^{\text {ell }}$ dell'ellissoide, è inferiore al valore della $g_{0}$ si della sfera; infatti le quattro grandezze sono legate dalla relazione di Gauss:

$$
4 \pi \varepsilon M=g_{0}{ }^{\mathrm{sf}} S^{\mathrm{sf}}=g_{0}{ }^{\mathrm{ell}} S^{\mathrm{ell}}
$$

e per essere $S^{\mathrm{ell}}>S^{\mathrm{sf}}$ è, di conseguenza, $g_{0}{ }^{\mathrm{ell}}<g_{\mathrm{o}}{ }^{\mathrm{sf}} ;$ precisamente è

$$
g_{\mathrm{o}}^{\mathrm{ell}} \sim \mathrm{g}_{\mathrm{o}}^{\mathrm{sf}}\left(1-\frac{8}{45} \alpha^{2}\right)
$$

Pertanto, nel passaggio considerato, l'energia diventa

$$
\begin{aligned}
W_{g 3} & =-\frac{3}{5} \frac{g_{0}{ }^{2} R^{3}}{\varepsilon}\left(1-\frac{16 \alpha^{2}}{45}\right)(1+\theta)^{3} \sim \\
& \sim \frac{3}{5} \frac{M^{2} \varepsilon}{R}\left(1-\frac{16 \alpha^{2}}{45}\right)\left(1-\theta+\theta^{2}\right)
\end{aligned}
$$

di conseguenza la variazione d'energia è

$$
\Delta W_{g}=W_{g 1}-W_{g 3}=W_{g 1}\left[\frac{16}{45} x^{2}-\theta+\theta^{2}\right]
$$


L'energia cinetica, al contrario, aumenta negli stessi passaggi e, detta $W_{c}$ quella della sfera iniziale cui si è comunicato (a spese dell'energia gravitazionale) la velocità angolare $W$, e $W^{\prime}$ quella dell'ellissoide finale, si ha

$$
W_{c}^{\prime}=W_{c}(1+20)\left(1+\frac{2 \alpha}{3}\right) \approx W_{\cdot}\left(1+21+\frac{2 \alpha}{3}\right)[\mathrm{g}]
$$

Per il principio di conservazione dell'energia deve essere

$$
W_{*}\left[\frac{16}{45} \alpha^{2}+\theta+\theta^{2}\right]=W .\left[1+2 \theta+\frac{2 \alpha}{3}\right]
$$

Possiamo ritenere $W_{c}$ infinitesimo del primo ordine rispetto a $W_{g}$ e i prodotti $\theta W_{c}, \propto W_{c}$ del secondo ordine, ne consegue che, trascurando in prima approssimazione $\mathrm{i}$ termini del secondo ordine, la [h] si riduce a $\theta W_{\mathbf{g}}=W_{c}$ e, sostituendo nella $[\mathrm{h}]$ stessa, si ricava appunto

$$
16 \alpha^{2}-30 \alpha \theta-135 \theta^{2}=0
$$

Per il calcolo di $W_{c}$ e $W_{g}$ bisognava fare alcune ipotesi sulla distribuzione interna della densità del geoide.

Nell'articolo Bernasconi C. (1974), per errore di calcolo, era riportato $W_{c}=209,0739$, da cui, correggendo, $\theta=0,838795.103, \alpha=3,3466066.10^{3}$.

Appare più razionale, capovolgendo il punto di vista allora adottato, determinare $\theta$ in funzione di $\alpha$ per avere poi elementi per uno studio sulla distribuzione della densità.

Posto dunque nella [5] $\alpha=1 / 297$, si ricava

$$
\theta=\frac{W_{c}}{W_{g}}=0,843911 \cdot 10^{-3}
$$

Si è ritenuto (in quanto è lecito assimilare il geoide ed una sfera per sviluppare ipotesi energetiche e gravitazionali), fosse più corretto calcolare l'energia cinetica alla sfera avente la stessa massa e velocità angolare del geoide. Con tale premessa il momento d'inerzia dalla sfera si deduce con sufficiente appros- 
simazione da quello del geoide (a sua volta approssimato all'ellissoide) mediante la relazione

$$
I_{p}^{s t}=I_{p} \frac{1-2 \alpha}{3}=7,91969 \cdot 10^{44}
$$

dove $I_{p}$ ha il valore numerico riportato nella [4] e di conseguenza

$$
\begin{gathered}
W_{c}=\frac{1}{2} I^{s t} \omega^{2}=209,41616 \cdot 10^{34} \\
W_{g}=248,14958 \cdot 10^{17}
\end{gathered}
$$

La $W_{g}$ si può dividere in due addendi: $W_{\mathrm{g}}{ }^{1}$ energia dovuta al campo gravitazionale interno al geoide, ed $W_{g}{ }^{e}$ dovuta al campo gravitazionale esterno.

Come noto si ha

$$
\begin{gathered}
W_{\mathrm{g}}^{c}=\frac{1}{2 \varepsilon} \int_{\mathrm{R}}^{\infty}\left(\frac{\varepsilon M}{r}\right)^{2} \mathrm{~d} r=\frac{1}{2} \frac{\varepsilon M^{2}}{R}=\frac{1}{2} \frac{g_{\mathrm{o}}{ }^{2} R^{3}}{\varepsilon}= \\
=186,3658 \cdot 10^{37}
\end{gathered}
$$

e pertanto

$$
W_{g i}=61.78378 \cdot 10^{37}
$$

Si hanno ora elementi per una impostazione puramente teoretica del problema della determinazione della densità $\delta(r)$ in funzione del raggio.

Il problema è matematicamente indeterminato, in quanto le condizioni cui deve soddisfare la $\bar{\partial}(r)$ non sono sufficienti, tuttavia sono bastevoli per interessanti congetture.

L'impostazione di tutti i sistemi trattati (risultata la più idonea) è stata mantenuta uguale a quella dell'esempio esposto e cioè con la caratteristica che il parametro a del secondo membro è il terzo nella progressione ordinata di ciascuna delle $\delta(r)$. 
Assumeremo $\delta(r)$ della forma

$$
\delta(r)=\delta_{0}+a_{1} x+a_{2} x^{2}+\ldots \ldots \ldots \ldots a_{\mathrm{n}} x^{\mathrm{n}}
$$

dove. ed $a_{1}$ sono costanti da determinare.

La gravità $g(r)$ è poi legata alla $\delta(r)$ dalla

$$
g(r)=\frac{4 \pi \varepsilon}{r^{2}} \int^{r} \delta(r) r^{2} \mathrm{~d} r
$$

La gravità $g(r)$ è poi legata alla $\delta(r)$ dalla

$$
\begin{gathered}
\frac{4}{3} \pi R^{3} \delta_{m}=M=4 \pi \int_{0}^{R} \delta(r) \dot{r} \mathrm{~d} r \\
I_{p}^{\mathrm{st}}=I=\frac{8 \pi}{3} \int_{0}^{R} \delta(r) r^{4} \mathrm{~d} r \\
W_{g, i}=\frac{\mathrm{i}}{2 \varepsilon} \int_{0}^{R} g^{2}(r) r^{2} \mathrm{~d} r \\
\delta(R)=\delta_{0}+a_{1}+a_{2}+\ldots \ldots \ldots . . .+a_{\mathrm{n}}
\end{gathered}
$$

quest'ultima rappresenta la densità media dello strato superficiale, profondo un centinaio di $\mathrm{Km}$.

Per comodità di calcolo porremo poi

$$
r=\frac{r}{10^{8}}, \quad R=\frac{R}{10^{8}}, \quad \varepsilon=\varepsilon \cdot 10^{8}
$$

Con tali sostituzioni si ha

$$
\delta(r)=\delta(r) ; \quad g(r)=g(r) ; I=\frac{I}{i \bar{U}^{i v}} ; W_{i}=\frac{W_{i}}{10^{32}}
$$


Tenendo presente la densità media del geoide $\delta_{m}=5,51472$ e i valori numerici di $I$ (vedi formula [7]) e di $W_{p t}$ (vedi formula [10]), ed esplicitando gli integrali, le [13] si traducono nelle:

$$
\begin{aligned}
& \frac{\delta_{0}}{3}+\frac{a_{1}}{4}+\frac{a_{0}}{5}+\frac{a_{0}}{6}+\ldots \ldots \ldots \ldots=1,838241417 \\
& \frac{\delta_{0}}{5}+\frac{n_{1}}{6}+\frac{n_{2}}{7}+\frac{a_{3}}{8}+\ldots \ldots \ldots . . . .=0,901348437 \\
& 1,112024587=\delta_{0}\left[\frac{\delta_{0}}{45}+\frac{a_{1}}{36}+\frac{a_{2}}{52,5}+\frac{a_{3}}{72}+\ldots \ldots \ldots \ldots\right]+ \\
& +a_{1}\left[\frac{a_{1}}{112}+\frac{a_{2}}{80} \div \frac{a_{3}}{108}+\ldots \ldots \ldots . .\right]+ \\
& +a_{2}\left[\frac{a_{2}}{225}+\frac{a_{2}}{150}+\ldots \ldots \ldots \ldots . .\right]+\ldots \ldots \ldots \\
& \delta_{0}+a_{1}+a_{2}+a_{3}+\ldots \ldots \ldots \ldots \ldots=3,5
\end{aligned}
$$

$E^{\prime}$ lecito ritenere che nel corso della sua evoluzione la Terra si sia assestata in modo che la sua energia potenziale sia la minima (ovvero massima in valore assoluto), pertanto il valore della $W_{g^{t}}$ riportato nella [13] è il massimo compatibile con gli elementi costituenti la Terra, la loro abbondanza percentuale e caratteristiche fisiche. Tale massimo si può ritrovare con infinite distribuzioni di densità tutte però monotone e decrescenti al crescere di $r$; la condizione di monotonia è già implicitamente contenuta nella formula dell'energia per cui risulta (entro certi limiti) automaticamente soddisfatta.

Delle infinite predette distribuzioni sarebbe inutile ricercare quella che fornisce il valore più alto di $\tilde{o}_{0}$ (densità al centro) essendo scontato che non esiste un limite superiore; infatti non è fisicamente assurdo pensare al centro della Terra, concentrata in piccolo volume, una massa di altissima densità.

$\mathrm{Ha}$ invece significato ricercare la distribuzione che fornisce 
il $\bar{\delta}_{\mathrm{o}}$ minimo, e in questo senso l'A. si è indirizzato studiando le distribuzioni del tipo:

$$
\begin{aligned}
& \delta(r)_{1}=\delta_{0}+a_{1} x+a_{2} x^{2}+a_{3} x^{3}+a_{4} x^{4} \\
& \delta(r)_{2}=\delta_{0}+a_{2} x^{2}+a_{3} x^{3}+a_{4} x^{4}+a_{5} x^{5} \\
& \delta(r)_{6}=\delta_{0}+a_{6} x^{6}+a_{7} x^{7}+a_{8} x^{8}+a_{9} x^{9}
\end{aligned}
$$

Ciascuna delle $\delta(r)$ contiene una costante in più delle condizioni; si è assunto come parametro la $\hat{b}_{0}$; di conseguenza ciascuna delle $a$ è funzione di $o_{0}$ che varia fra un massimo e un minimo. L'interesse si appunta sul minimo, ma è anche significativo vedere se queste distribuzioni si adattano alle distribuzioni teoriche o semiempiriche già note.

E' chiaro che il massimo di $\delta_{0}$ si abbassa da $\delta(r)_{1}$ a $\delta(r)_{6}$ e che man mano si estende una sorta di pianerottolo a partire dall'origine; è altresì intuitivo che il pianerottolo debba a un certo punto flettere più o meno bruscamente; la collocazione del flesso può aiutare nell'interpretazione fisica.

Come esempio di calcolo viene riportata la determinazione di $\delta(r)_{6}$.

Si inizia col risolvere il sistema

$$
\begin{aligned}
\frac{a_{6}}{9}+\frac{a_{8}}{11}+\frac{a_{9}}{12} & =1,8382414-\frac{\delta_{0}}{3}-\frac{a_{7}}{10} \\
\frac{a_{6}}{11}+\frac{a_{8}}{13}+\frac{a_{9}}{14} & =0,901348-\frac{\delta_{0}}{5}-\frac{a_{7}}{12} \\
a_{6}+a_{8}+a_{9} & =3,5-\delta_{0}-a_{7} \\
11,2 & \leq \delta_{0} \leq 11,8
\end{aligned}
$$

rispetto ad $a_{6}, a_{8}, a_{9}$ in funzione di $\delta_{0}$ e di $a_{7}$; sostituendo poi nella [16] si ricava una equazione di $2^{\circ}$ grado dalla quale si 
deduce $a_{7}$ in funzione di $\partial_{0}$. La condizione di realtà delle radici impone che il discriminante dell'equazione sia positivo e quindi determina il campo di variabilità di $\delta_{o}$; nel caso nostro risulta

$$
-1,290718 \leq \bar{\delta}_{o} \leq 12,112567 \text {. }
$$

Il campo di variabilità fisico, nel quale la funzione si mantiene monotona, è

$$
\sim 11,2 \leq \delta_{0} \leq \sim 11.8
$$

Assegnato a $\delta_{\mathrm{o}}$ un valore compreso fra 11,2 e 11,8 si trovano due valori di $a_{7}$, dei quali si elimina il più alto in valore relativo, e, successivamente si trovano i valori di $a_{6}, a_{8}, a_{9}$.

Si sono riportati nella tabella 1 gli estremi del campo di variabilità matematico e fisico di $\partial_{0}$ nelle funzioni $\tilde{\partial}(r)_{\mathrm{i}}$

TABELLA 1

\begin{tabular}{|c|c|c|c|c|}
\hline funzioni & $\begin{array}{c}\hat{o}_{0} \mathrm{~min} \\
\text { (matem.) }\end{array}$ & $\begin{array}{c}\tilde{o}_{0} \mathrm{~min} \\
\text { (fisico) }\end{array}$ & $\begin{array}{c}\tilde{\delta}_{0} \mathrm{mx} \\
\text { (matem.) }\end{array}$ & $\begin{array}{c}\tilde{\delta}_{0} \mathrm{mx} \\
\text { (fisico) }\end{array}$ \\
\cline { 1 - 2 }$\delta(r)_{1}$ & $-0,4174$ & 14,50 & 25,8353 & 25,80 \\
$\delta(r)_{2}$ & $-0,6623$ & 13,00 & 18,0527 & 18,00 \\
$\delta(r)_{3}$ & $-1,01351$ & 12,50 & 15,1379 & 15,10 \\
$\delta(r)_{4}$ & $-1,3712$ & 12,00 & 13,9394 & 13,90 \\
$\delta(r)_{5}$ & $-1,3026$ & 11,40 & 13,2529 & 12,60 \\
$\delta(r)_{6}$ & $-1,2907$ & 11,20 & 12,1125 & 11,80 \\
\end{tabular}

Riportando in ordinata, in funzione dell'indice $1,2, \ldots, 6$, il minimo e il massimo di $\tilde{\partial}_{0}$, si ottengono due curve che, per estrapolazione, si intersecano in $\bar{o}_{0} \sim 10,8$, valore che appare come il minimo della densità al centro del geoide.

Riportiamo nella tabella 2 alcune serie di valori di $\delta(r)_{t}$ per $r=R^{n} / 10$, con $n=0,1,2, \ldots, 10$, scegliendo quelle nelle quali il flesso si trova all'incirca per $n=5,447$, ossia a $2.900 \mathrm{Km}$ di profondità dalla crosta terrestre. Sembrerebbe da questo studio, 
puramente matematico, che il valore più probabile di $\delta_{0}$, tenendo conto della condizione relativa al flesso, debba collocarsi tra 12,5 e 14.

TABELLA 2

\begin{tabular}{|c|c|c|c|c|c|c|c|c|c|}
\hline \multicolumn{2}{|r|}{$\delta(r)_{2}$} & \multicolumn{2}{|r|}{$\tilde{\partial}(r)_{3}$} & \multicolumn{2}{|r|}{$\delta(r)_{4}$} & \multicolumn{2}{|r|}{$\tilde{\partial}(r)_{4}$} & \multicolumn{2}{|r|}{$\tilde{\delta}(r)_{5}$} \\
\hline$\tilde{\partial}_{0}$ & 14,0000 & $\hat{\partial}_{2}$ & 13,0000 & $\delta_{0}$ & 13,9000 & $\delta_{0}$ & 13,0000 & $\delta_{0}$ & 12,5000 \\
\hline$a_{2}$ & $-27,1910$ & $a_{3}$ & $-73,8005$ & $a_{4}$ & $-319,0976$ & $a_{4}$ & $-211,2723$ & $a_{5}$ & $-382,1627$ \\
\hline$a_{3}$ & 9,7135 & $a_{4}$ & 104,8190 & $a_{5}$ & 728,0846 & $a_{5}$ & 418,1217 & $a_{6}$ & 806,5351 \\
\hline$a_{4}$ & $-6,6455$ & $a_{6}$ & -48.0310 & $a_{6}$ & $-565,3436$ & $a_{6}$ & $-264,9245$ & $a_{7}$ & $-525,3168$ \\
\hline$a_{5}$ & 0,3319 & $a^{6}$ & 7,5124 & $a_{7}$ & 145,9566 & $a_{7}$ & 48,5751 & $a_{8}$ & 91,9444 \\
\hline 0 & 14,0000 & 0 & 13,0000 & 0 & 13,9000 & 0 & 13,0000 & 0 & 12,5000 \\
\hline 1 & 13,7384 & 1 & 12,9362 & 1 & 13,8748 & 1 & 12,9827 & 1 & 12,4969 \\
\hline 2 & 13,0008 & 2 & 12,5624 & 2 & 13,5881 & 2 & 12,7794 & 2 & 12,4228 \\
\hline 3 & 11,8697 & 3 & 11,7451 & 3 & 12,7043 & 3 & 12,1222 & 3 & 12,0504 \\
\hline 4 & 10,4446 & 4 & 10,4991 & 4 & 11,1101 & 4 & 10,8674 & 4 & 11,0897 \\
\hline 5 & 8,8421 & 5 & 8,9425 & 5 & 9,0158 & 5 & 9,1018 & 5 & 9,4146 \\
\hline 6 & 7,1964 & 6 & 7,2592 & 6 & 6,8699 & 6 & 7,1317 & 6 & 7,2515 \\
\hline 7 & 5,6595 & 7 & 5,6647 & 7 & 5,1618 & 7 & 5,3795 & 7 & 5,1962 \\
\hline 8 & 4,4018 & 8 & 4,3785 & 8 & 4,1842 & 8 & 4,2115 & 8 & 3,9600 \\
\hline 9 & 3,6124 & 9 & 3,6018 & 9 & 3,8305 & 9 & 3,7225 & 9 & 3,7842 \\
\hline 10 & 3,5000 & 10 & 3,5000 & 10 & 3,5000 & 10 & 3,5000 & 10 & 3,5000 \\
\hline
\end{tabular}

La discontinuità a $2.900 \mathrm{Km}$ di profondità, sperimentalmente riconosciuta, è ritenuta di diversa ampiezza in particolare da Bullen e Bullard. Sul testo B. Gutenberg, 1959 è inclusa, a pag. 155 una tavola che riporta i valori della densità e gravità in funzione del raggio secondo gli Autori sopracitati. In base a tali valori è agevole calcolare il momento d'inerzia e la energia interna che risultano.

$$
\begin{array}{lll}
I_{\mathrm{p}}=8,3358 \cdot 10^{44} & W_{R i}=61,4788 \cdot 10^{37} & \text { (modello Bullen) } \\
I_{\mathrm{p}}=8,3358 \cdot 10^{44} & W_{k i}=61,24212 \cdot 10^{37} & \text { (modello Bullard) }
\end{array}
$$




\section{BIBLIOGRAFIA}

Bernasconi C., 1974 - Determinazione teorica dello schiacciamento dello sferoide terrestre. "Boll. Ass. Geof. It. », XXIII, 3-4, 149-153.

Gutemberg B., 1959 - Physics of the Earth Inferior. "Academic Press", New York, London. 